\title{
An Arabidopsis Cytokinin-Modifying Glycosyltransferase UGT76C2 Improves Drought and Salt Tolerance in Rice
}

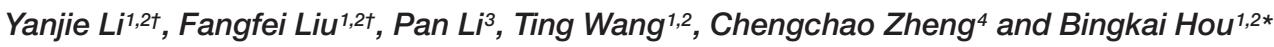 \\ 'The Key Laboratory of Plant Development and Environment Adaptation Biology, Ministry of Education, Shandong \\ University, Qingdao, China, ${ }^{2}$ School of Life Sciences, Shandong University, Qingdao, China, ${ }^{3}$ College of Pharmacy, \\ Liaocheng University, Liaocheng, China, ${ }^{4}$ State Key Laboratory of Crop Biology, College of Life Sciences, Shandong \\ Agricultural University, Taian, China
}

OPEN ACCESS

Edited by:

Klára Hoyerová

Academy of Sciences of the Czech

Republic, Czechia

Reviewed by:

Aaron M. Rashotte,

Auburn University, United States

Marketa Pernisova,

Masaryk University, Czechia

Karel Dolezal,

Academy of Sciences of the Czech

Republic, Czechia

*Correspondence:

Bingkai Hou

bkhou@sdu.edu.cn

${ }^{t}$ These authors have contributed

equally to this work

Specialty section:

This article was submitted to

Plant Physiology,

a section of the journal

Frontiers in Plant Science

Received: 15 June 2020

Accepted: 09 October 2020

Published: 05 November 2020

Citation:

Li Y, Liu F, Li P, Wang T, Zheng C

and Hou B (2020) An Arabidopsis

Cytokinin-Modifying

Glycosyltransferase UGT76C2 Improves Drought and Salt Tolerance in Rice. Front. Plant Sci. 11:560696.

doi: 10.3389/fpls.2020.560696
Drought and salt stresses are common environmental threats that negatively affect rice development and yield. Here we report that the overexpression of AtUGT76C2, a cytokinin glycosyltransferase, in rice modulates cytokinin homeostasis and confers the plants an eminent property in drought and salt tolerance. The transgenic plants exhibit sensitivity to salt and drought stress as well as abscisic acid during the germination stage and the postgermination stage while showing enhanced tolerance to drought and salinity at the young seedling stage and the mature stage. The overexpression of UGT76C2 decreases the endogenous cytokinin level and enhances root growth, which greatly contributes to stress adaptation. In addition, the transgenic plants also show enhanced ROS scavenging activity, reduced ion leakage under salt stress, smaller stomatal opening, and more proline and soluble sugar accumulation, which demonstrate that UGT76C2 acts as an important player in abiotic stress response in rice. To explore the molecular mechanism of UGT76C2 in response to stress adaptation, the expressions of eight stress-responsive genes including OsSOS1, OsPIP2.1, OsDREB2A, OsCOIN, OsABF2, OsRAB16, OsP5CR, and OsP5CS1 were detected, which showed notable upregulation in UGT76C2 overexpression plants under salt and drought stresses. Our results reveal that the ectopic expression of AtUGT76C2 confers the transgenic rice many traits in improving drought and salt stress tolerance in both developmental and physiological levels. It is believed that AtUGT76C2 could be a promising candidate gene for cultivating saline- and drought-tolerant rice.

Keywords: abscisic acid, AtUGT76C2, drought stress, salt stress, glycosyltransferase, rice

\section{INTRODUCTION}

Drought and salinity are the most common abiotic threats limiting plant growth and crop yield. As the most consumed staple food in the world, rice is extremely sensitive to these stresses. Rice requires sufficient water during the whole life cycle, especially at the grain filling stage. Until now, drought and salinity have become major factors causing rice yield loss. Thus, a study exploring 
critical saline- and drought-tolerant genes as well as revealing their molecular mechanisms is a critical prerequisite for cultivating saline- and drought-tolerant rice via molecular design breeding.

Until now, many stress-regulated genes have been identified to improve stress resistance in plants, which can be grouped into four types: genes involved in the synthesis of osmotic regulators, such as proline biosynthesis gene OsP5CS1 and OsP5CR ( Hu et al., 1992; Sripinyowanich et al., 2013); genes involved in ion transportation, such as SOS1 (Shi et al., 2000); antioxidant-related genes, such as CAT (catalase), APX (ascorbate peroxidase), and SOD (superoxide dismutase) (Mhamdi et al., 2010; Verma et al., 2019); and genes regulating signaling cascades, such as protein kinases (Kang et al., 2017), transcription factors, and so on (Xiong et al., 2014; Chen et al., 2015). However, to enrich the present knowledge and uncover the molecular mechanism on stress regulation in every aspect, more pathways still need to be elucidated.

Cytokinins (CK) are N6-substituted adenine derivatives which are originally defined as a key regulator participating in various plant developmental activities, including organ formation, apical dominance, and leaf senescence (El-Showk et al., 2013). Nowadays, increasing evidence also indicates the action of cytokinin in response to stresses (Hai et al., 2020). A recent cytokinin-responsive transcriptome analysis in rice revealed that a large number of genes are involved in both biotic and abiotic stresses (Raines et al., 2016). It is also reported that cytokinin is required for responding to a series of environmental factors including temperature, drought, osmotic stress, salinity, nutrient stress, plant pathogens, and herbivores (Raines et al., 2016; Cortleven et al., 2019). Genetic studies by modifying cytokinin level in plants indicate that it generally serves as a negative player in stress response (Nishiyama et al., 2011). For instance, the overexpression of the cytokinin-degrading enzyme cytokinin oxidase/dehydrogenase gene enhanced the drought and heat stress tolerance in transgenic tobacco plants (Macková et al., 2013). Previous studies found that AHK2, AHK3, and AHK4 are negative regulators of dehydration and salt tolerance, and the $a h k 2$ and $a h k 3$ mutants displayed higher survival rates under severe water deprivation (Tran et al., 2007; Kang et al., 2012). However, some studies found that cytokinin could also function as a positive regulator in drought stress adaptation (Hai et al., 2020). For example, the ectopic expression of the isopentenyltransferase gene (IPT) that encodes a rate-limiting enzyme in cytokinin biosynthesis increases endogenous cytokinin levels as well as improves drought stress tolerance in transgenic cotton (Kuppu et al., 2013), creeping bentgrass (Xu et al., 2016), eggplant (Xiao et al., 2017), and tropical maize (Leta et al., 2016). It might be attributed to the choice of promoter, which led to the regulation of different pathways.

Plants tend to fine-tune the developmental process and environmental responses via crosstalk between phytohormones. A number of studies have revealed the interplay between cytokinin and abscisic acid (ABA), which act antagonistically in regulating stress response. In contrast to cytokinin, ABA synthesis will be induced in response to water loss, which represents a protective role in response to adverse conditions via arresting seed germination and postgermination growth (Lopez-Molina et al., 2002), modulating stomata closure (Zhang et al., 1987) as well as regulating the expression of stressresponsive genes (Zhu, 2002). An earlier study found that transzeatin riboside decreases significantly in sunflower plants upon exposure to drought stress, while the endogenous ABA content was increased (Hansen and Dörffling, 2003). Jeon et al. (2010) reported that $\mathrm{AHK} 2, \mathrm{AHK} 3$, and A-type ARRs act as negative regulators in cold stress signaling pathway via inhibiting ABA response. It is illustrated that both the ahk2 ahk3 double mutant and the $\operatorname{arr} 7$ single mutant showed enhanced freezing tolerance, while they are hypersensitive to ABA. Although cytokinin and ABA antagonistically regulate many processes, the molecular mechanisms are poorly known. Recently, Huang et al. (2018) reported that the ABA-activated SnRK2s could phosphorylate the negative regulator type-A response regulator 5 and enhance its stability upon drought stress, by which amplifying the ABAmediated stress response. This study provides insights into the molecular mechanism of how ABA and cytokinin interplay antagonistically in response to stress.

The levels of endogenous cytokinin is closely related to small molecular modification by the family 1 UDP glycosyltransferases (UGTs), which transfer sugar moieties to small acceptor molecules (Bowles et al., 2006; Lairson et al., 2008). The Arabidopsis UGT76C1 and UGT76C2 have been identified to finely tune the glycosylation of cytokinins, which deactivate the molecule and play crucial roles in regulating cytokinin homoestasis in plants (Hou et al., 2004). In our previous study, we revealed that $U G T 76 C 2$ is repressed by $\mathrm{ABA}$, osmotic stress, and drought stress. The ectopic expression of UGT76C2 led to sensitivity to $\mathrm{ABA}$ and mannitol during germination stage and tolerance to drought stress as established big plants. It is typically an ABA-dependent pathway in resisting abiotic stresses. We demonstrated that the involvement of UGT76C2 to water deprivation response can be mediated via cytokinin and $\mathrm{ABA}$ correlations in Arabidopsis (Li et al., 2015).

Many identified stress-regulated UGTs were also heterologously expressed in other plant species and bring special properties to the transgenic plants. For example, the ectopic expression of the Arabidopsis UGT85A5 in transgenic tobacco enhanced salt tolerance in the plants (Sun et al., 2013). Li et al. (2017) found that a barley UDP-glucosyltransferase HvUGT13248, which modify deoxynivalenol and nivalenol, provides Fusarium head blight resistance in transgenic wheat. Although a number of rice UGTs are putatively stress-responsive upon examination on Genevestigator ${ }^{1}$, no stress-related UGTs have been functionally characterized in rice, and none of the known UGTs have been introduced into rice yet. To see what effect will a stress-related UGT bring to rice, here we transferred the Arabidopsis UGT76C2 into rice and further explored its role. Our results showed that AtUGT76C2 greatly enhanced the plant tolerance to drought and salt in transgenic rice in both developmental and physiological levels. This study laid a theoretical foundation that UGT76C2 could be a promising

\footnotetext{
${ }^{1}$ https://genevestigator.com/
} 
candidate gene for cultivating saline- and drought-tolerant plants in both dicots and monocots.

\section{MATERIALS AND METHODS}

\section{Plant Materials, Vector Construct, and Plant Transformation}

Oryza japonica was used in this study. Seedlings were grown on MS plates or soil under SD (10 h light/14 h dark) condition at $28^{\circ} \mathrm{C}$ in a growth chamber. To generate UGT76C2 overexpression vector driven by a ubiquitin promoter, the coding region of Arabidopsis UGT76C2 (At5g05860) was PCR-amplified and sequenced to be right. Then, the fragment was inserted into pUN1301 binary vector by KpnI and BamHI digestion and $\mathrm{T}_{4}$ ligation. Rice transformation was performed by Biorun biological company ${ }^{2}$.

\section{HPLC and LC-MS Analysis}

For analyzing the reaction activity toward cytokinin, total protein was extracted from wild-type (WT), OE2, and OE41 plants. The glycosyltransferase activity assay toward trans-zeatin was carried out with $0.1 \mathrm{mg}$ total protein, $1 \mathrm{mM}$ trans-zeatin, $5 \mathrm{mM}$ UDPglucose, $50 \mathrm{mM}$ HEPES (pH 7.0), $2.5 \mathrm{mM} \mathrm{MgSO}_{4}, 10 \mathrm{mM} \mathrm{KCl}$, and $14.4 \mathrm{mM} \beta$-mercaptoethanol in $100 \mu \mathrm{l}$ reaction mix. The reaction mix was incubated at $30^{\circ} \mathrm{C}$ for $3 \mathrm{~h}$. The total protein was extracted in three biological replicates. For analyzing cytokinin glycosides produced in the plants, 7-day-old WT, UGT76C2OE2, and UGT76C2OE41 were treated with $150 \mu \mathrm{mol}$ trans-zeatin for $24 \mathrm{~h}$, and the total glycosides were extracted with $80 \%$ methanol. The treatment and the total glycoside extraction were done in three biological replicates. High-performance liquid chromatography (HPLC) was performed on a Shimadzu HPLC system (Japan). Then, a $10 \mu$ l-sample was loaded onto a $5-\mu \mathrm{m}$ C18 column (150 mm $\times 4.6 \mathrm{~mm}$; Welch, Ultimate). A linear gradient with increasing methanol (solvent $\mathrm{A}$ ) against $0.1 \%$ triethylamine acetate (solvent $\mathrm{B}$ ) at a flow rate of $1 \mathrm{ml} / \mathrm{min}$ over 40 min was used. Both solutions contained $0.1 \% \mathrm{H}_{3} \mathrm{PO}_{4}$. The peak of trans-zeatin was monitored at $245 \mathrm{~nm}$. For liquid chromatography-mass spectrometry (LC-MS) analysis (Shimadzu), the methods and the mobile phases were similar to the HPLC condition. The mass spectrometer was operated in a positive electrospray ionization mode with $50 \mathrm{eV}$ and a probe voltage of $5.0 \mathrm{kV}$. The dry heater was set to $180^{\circ} \mathrm{C}$. Data acquisition and analysis were performed with Xcalibur software (version 2.0.6).

\section{Stress Assays}

For calculating seed germination rates under stresses, full and same-size rice seeds were surface-sterilized in $75 \%$ ethanol for $2 \mathrm{~min}$ and then in $0.1 \%$ mercuric chloride solution for $3 \mathrm{~min}$ and rinsed three or four times. The sterilized seeds were imbibed at $28^{\circ} \mathrm{C}$ in the presence of water (control) and a hydroponic solution of $100 \mathrm{mM} \mathrm{NaCl}, 7.5 \%$ PEG8000, and $150 \mathrm{mM}$ mannitol. For the ABA treatment, ABA was firstly dissolved in a small

${ }^{2}$ http://www.biorun.net/ amount of ethanol and then diluted into the hydroponic solution with 2 and $5 \mu \mathrm{M}$ ABA. The seeds were germinated in SD (10 $\mathrm{h}$ light $/ 14 \mathrm{~h}$ dark) condition at $28^{\circ} \mathrm{C}$ in a growth chamber and were regarded as germinated when the radicles protrude from the seed coat over $2 \mathrm{~mm}$. For each replicate, 50 seeds were calculated. The subsequent seedling growth was observed 5 days later and photographed. For drought stress treatment, watering of 2-week-old rice seedlings growing in soil was stopped until they wilted, followed by re-irrigation. After recovery, the performance of the plants in stress tolerance was observed and photographed. For salt stress, the WT and overexpression lines were irrigated with $200 \mathrm{mM} \mathrm{NaCl}$ until they show differences in stress tolerance. Each stress treatment experiment contained three biological replicates. After the treatment, the survival rate was calculated. The recovered plants were regarded as survivors.

\section{Activities of ROS-Scavenging Enzymes}

The leaves were detached from 1-week-old rice plants after $200 \mathrm{mM} \mathrm{NaCl}$ and 15\% PEG8000 treatments for $12 \mathrm{~h}$, and the activities of ROS-scavenging enzymes such as APX, CAT, and SOD activities were determined according to a previously described method (Cai et al., 2015). In this assay, three independent samples were collected, and three technical replicates were done for evaluating enzyme activities.

\section{Diaminobenzidine and Nitrobluetetrazolium Staining, Determination of $\mathrm{H}_{2} \mathrm{O}_{2}$ Content}

For the determination of $\mathrm{H}_{2} \mathrm{O}_{2}$ and superoxide accumulation under abiotic stress conditions, 4-week-old rice plants were exposed to $200 \mathrm{mM} \mathrm{NaCl}$ and $15 \%$ PEG8000 for $24 \mathrm{~h}$, respectively, and then were harvested for diaminobenzidine $(\mathrm{DAB})$ and nitroblue tetrazolium (NBT) staining. Briefly, the leaves were firstly infiltrated by vacuum for $1 \mathrm{~h}$ and then subjected to $0.1 \% \mathrm{DAB}$ staining for $24 \mathrm{~h}(\mathrm{pH}=5.8)$, followed by incubation in the de-staining buffer (ethanol/lactic acid/glycerol $=3: 1: 1$ ). $\mathrm{DAB}$ staining was performed according to Du et al. (2008) to determine the $\mathrm{H}_{2} \mathrm{O}_{2}$ content. NBT staining for superoxide detection was conducted as described by Wohlgemuth et al. (2002). At least five independent plants were subjected to staining for each line. To determine the content of $\mathrm{H}_{2} \mathrm{O}_{2}$, Catalase Assay Kit (S0051, Beyotime, Shanghai, China) was used according to the user's manual. In brief, the 4-week-old rice plants were treated under salt and drought stress for $24 \mathrm{~h}$, and $0.1 \mathrm{~g}$ of tissue was harvested and homogenized with reagents in the kit. Being catalyzed by the catalase, the accumulated $\mathrm{H}_{2} \mathrm{O}_{2}$ was changed into a red product that can be determined by a spectrophotometer at $\mathrm{A}_{520}$. In the assay, $\mathrm{H}_{2} \mathrm{O}_{2}$ was determined at three biological replicates.

\section{Determination of Cytokinin Content}

To determine the cytokinin content, $0.1 \mathrm{~g}$ of fresh leaves of different lines were weighed, and for each line, three independent samples were collected as three biological replicates. The leaf tissues were homogenized in $900 \mu \mathrm{l}$ phosphate-buffered saline (PBS; pH 7.2-7.4) and centrifuged for $20 \mathrm{~min}$ at the speed of 
3,000 rpm, and then the supernatant was collected. For the quantification of cytokinin, ELISA Kit (MM-3259201) ${ }^{3}$ was used. The concentration of cytokinin in the samples is determined by a microplate reader (Tecan, InfiniteTMM200 PRO).

\section{Determination of Proline and Soluble Sugar Content}

To determine the content of proline and soluble sugar, 4week-old rice plants growing under normal condition were treated with $200 \mathrm{mM} \mathrm{NaCl}$ and 15\% PEG8000 for $24 \mathrm{~h}$, respectively, and the untreated plants served as control. For each line, three independent samples with $0.1 \mathrm{~g}$ harvested leaf tissues were collected. The samples were homogenized in 3\% aqueous sulphosalicylic acid and centrifuged. The supernatant was collected, and equal volumes of glacial acetic acid and ninhydrin were added. The content of proline was determined at $520 \mathrm{~nm}$ (Nakazawa et al., 1982). To examine the content of soluble sugar, $0.1 \mathrm{~g}$ of harvested leaves was crushed into powder in liquid nitrogen and homogenized in $80 \%$ ethanol, followed by incubation at $95^{\circ} \mathrm{C}$ for $1 \mathrm{~h}$. The supernatant was collected by centrifugation, dried at $80^{\circ} \mathrm{C}$ for $2 \mathrm{~h}$, and then dissolved in $10 \mathrm{ml}$ distilled water. Quantification of soluble sugar was performed as described by Li et al. (2004).

\section{Calculations on Ion Leakage, Water Loss, and Water Content of Detached Leaves}

Upon exposure to $200 \mathrm{mM} \mathrm{NaCl}$ for 7 days, rice leaves were collected from 2-week-old rice seedlings to measure the electrolyte leakage as described by Tang et al. (2017). For calculating water loss, fresh leaves detached from 3-week-old plants were firstly weighed (FW) and then dried naturally in open air. The drying leaves were weighed (DW) every $30 \mathrm{~min}$. Water loss was calculated as (FW - DW)/time * FW. For each assay, three biological replicates were performed.

\section{Observations of Stomatal Opening by Scanning Electron Microscopy}

Leaves of 3-week-old UGT76C2 transgenic rice and WT plants were detached and kept in air for $2 \mathrm{~h}$ to allow water loss and stimulate stomatal closure; then, the leaf tissues were fixed in $2.5 \%$ glutaraldehyde at $4^{\circ} \mathrm{C}$ for $12 \mathrm{~h}$ and then washed five times with $0.1 \mathrm{~mol} / \mathrm{L}$ phosphate buffer, followed by dehydration with gradient ethanol. The samples were bonded with a drying instrument on the HCP-2 critical point, followed by spraying gold with an IB-v ion sputtering device and photographed with a scanning electron microscope (FEI Quanta250 FEG). For each line, at least 100 stomata were observed.

\section{Determination of ABA Content}

Leaves of 3-week-old UGT76C2 transgenic rice and WT plants were detached and kept in air for $2 \mathrm{~h}$. For each line, three independent samples were collected, at approximately $50 \mathrm{mg}$ leaf tissue for each sample. The samples were rapidly frozen

${ }^{3}$ http://www.mmbio.cn/ with liquid nitrogen, followed by homogenization in PBS ( $\mathrm{pH}$ 7.4). The samples were centrifuged for $20 \mathrm{~min}$, and the supernatant was collected carefully for determination. The ABA concentration was assayed according to the instructions provided by the plant hormone abscisic acid ELISA Kit (Shanghai Fusheng Industrial Co., Ltd., catalog number: A112641-96T).

\section{Quantitative and Semi-Quantitative RT-PCR}

For quantitative and semi-quantitative RT-PCR, total RNA was extracted from rice samples with Trizol reagent (Vazyme), and 5 $\mu \mathrm{g}$ RNA was reverse-transcribed with the PrimeScript RT reagent kit with gDNA Eraser (Vazyme) according to the supplier's manual. Real-time PCR was done with a real-time thermal cycling system (Bio-Rad). SYBR-Green was used to detect gene abundances. Each reaction was done with three biological replicates. Data were analyzed using Bio-Rad CFX Manager software. For evaluating UGT76C2 expression in transgenic rice, semi-quantitative RT-PCR was employed. The synthesized cDNA was diluted five times (1:5), and $2 \mu \mathrm{l}$ was used for analyzing the transcript level. OsActin1 was used as an internal reference gene. PCR reactions were performed in $25 \mu \mathrm{l}$ of total reaction volume, with 25 cycles for amplifying OsActin 1 and 32 cycles for amplifying $U G T 76 C 2$. The primers for the RT-PCR assay are included in Supplementary Table S1.

\section{Statistical Analysis}

Student's $t$-test with one-tailed test was performed to compare the performance of WT and UGT76C2 transgenic lines. For each assay and experiment, data from at least three biological replicates were subjected to statistical analysis. The minimum significance levels were set at ${ }^{*} p<0.05$ and ${ }^{* *} p<0.01$.

\section{RESULTS}

\section{Overexpression of the Arabidopsis UGT76C2 in Rice Generates More Cytokinin Glycosides}

To determine the role of the Arabidopsis UGT76C2 in rice, transgenic plants overexpressing AtUGT76C2 under the control of the maize ubiquitin (ubi) promoter were generated, and the homozygous lines were selected with hygromycin. qRT-PCR analysis indicated that the transcripts of AtUGT76C2 could be detected in transgenic plants, but not in WT plants (Figure 1A). The UGT76C2 overexpression lines OE2 and OE41 with relatively higher UGT76C2 abundances were chosen for all assays in this study. To determine the role of UGT76C2 in catalyzing cytokinins in rice, firstly, we purified the total protein from WT, OE2, and OE41 plants and assessed their activity in catalyzing trans-zeatin. It was found that more trans-zeatin-7-N-Glc and trans-zeatin-9-N-Glc were produced after incubation with total protein purified from OE2 and OE41 than that from WT (Figures 1B1-3). The identification of 7 and 9-N-Glc was based on their retention time as reported by Hou et al. (2004) and by LC-MS confirmation (Figure 1C). To further investigate 


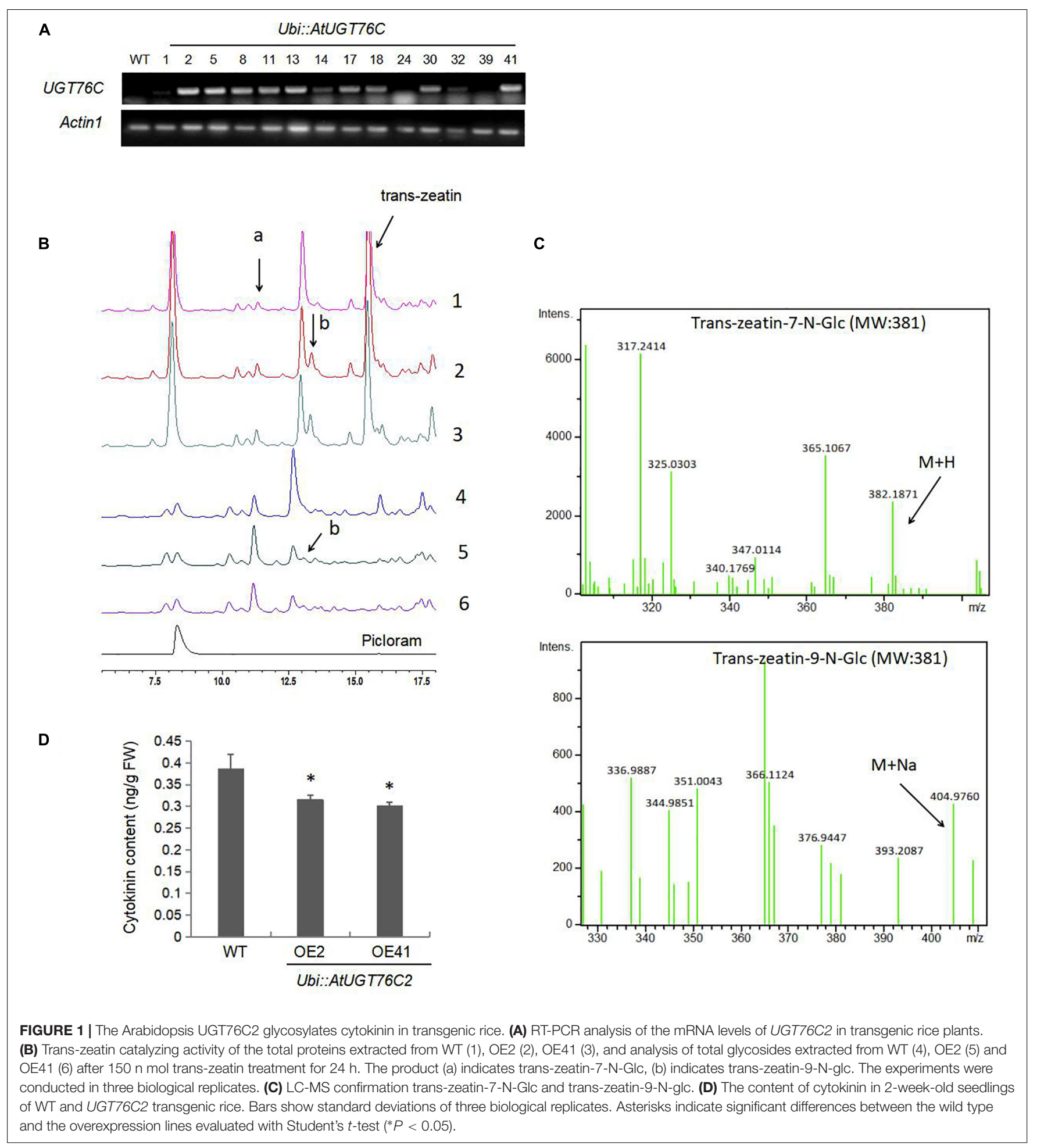

UGT76C2 activity toward trans-zeatin in rice, 7-day-old WT, UGT76C2OE2, and UGT76C2OE41 seedlings were treated with $150 \mu \mathrm{mol}$ trans-zeatin for $24 \mathrm{~h}$. Then, total glycosides were extracted from the three plants and subjected to HPLC and LC-MS analysis. Consistent with a previous study (Hou et al., 2004), more 7-N-Glc was generated than 9-N-Glc in plants
(Figures 1B4-6), which further demonstrates that UGT76C2 has a higher activity toward 7-N of trans-zeatin. However, for the total protein-catalyzed reaction in vitro, it seems that more 9-N-Glc was produced than 7-N-Glc (Figures 1B1-3). It is likely that UGT76C2 activity is affected by other proteins in the mix. These results showed that UGT76C2 overexpression in 
A
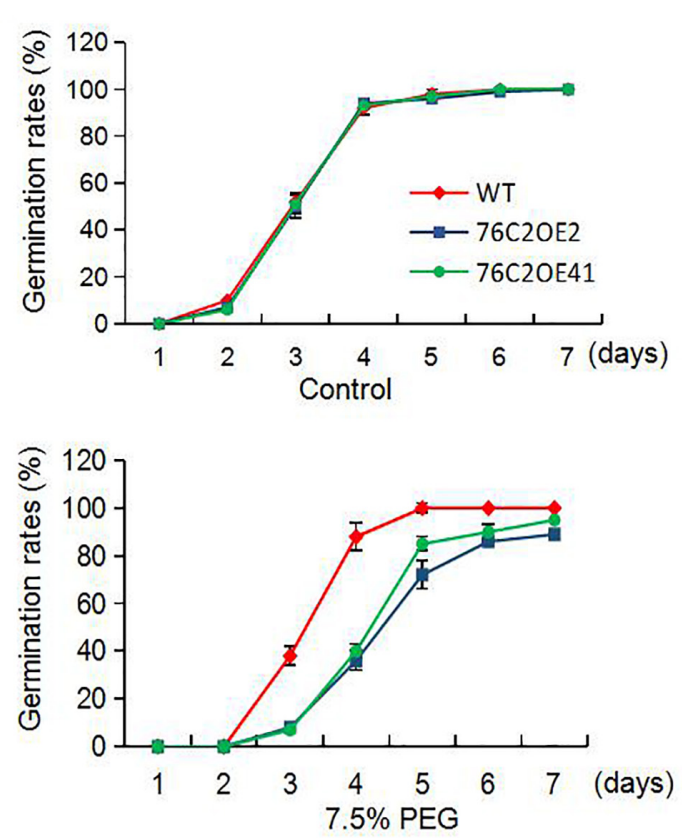
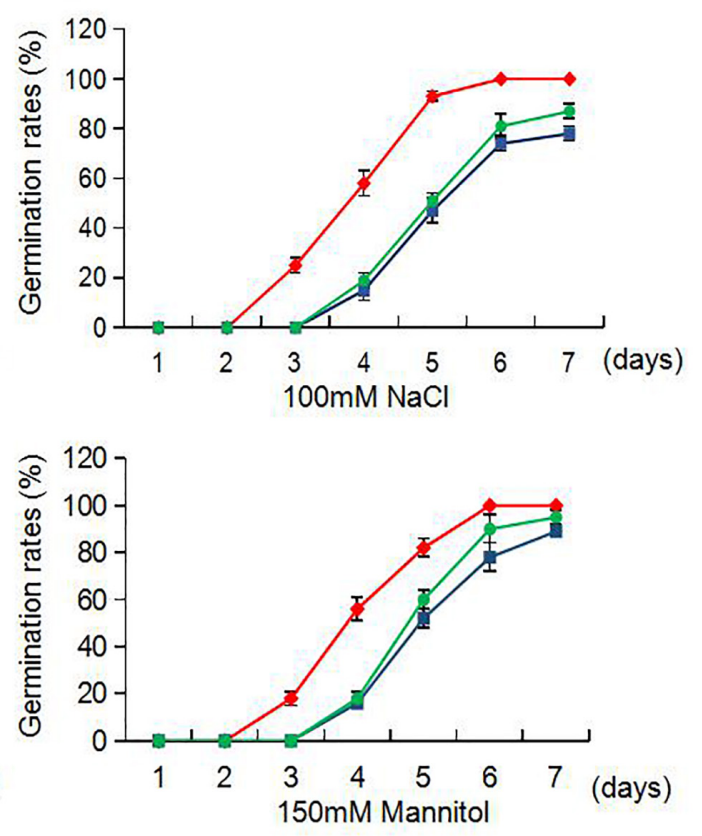

B WT 76C2OE2 76C2OE41
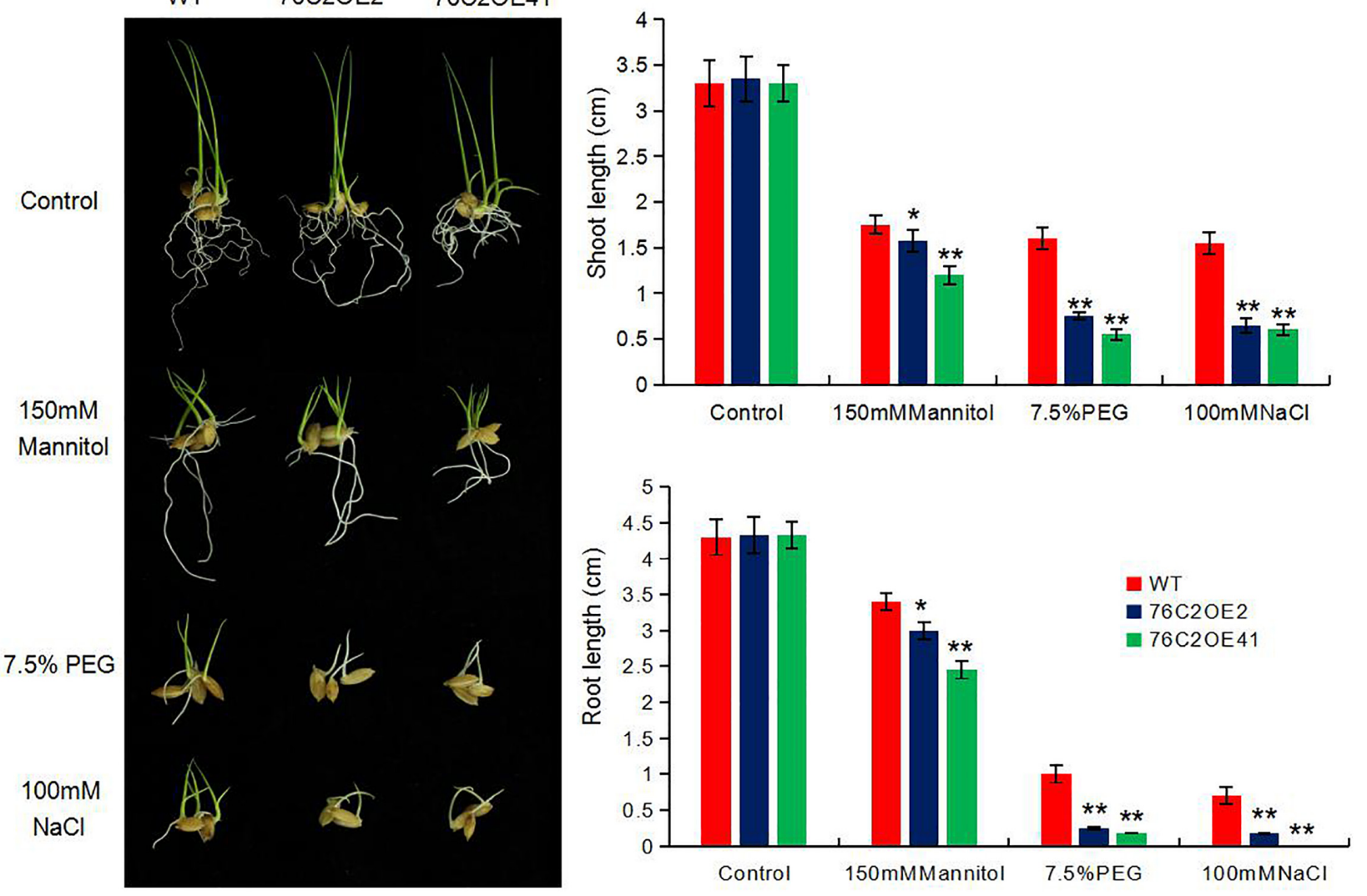

FIGURE 2 | Overexpression of AtUGT76C2 increased the plant sensitivity to abiotic stresses during germination and post-germination growth. (A) Rice seeds were imbibed at $28^{\circ} \mathrm{C}$ in the presence of water (Control) and hydroponic solution of $100 \mathrm{mM} \mathrm{NaCl}, 7.5 \%$ PEG and $150 \mathrm{mM}$ mannitol. Germination (based on radicles $>2$ $\mathrm{mm}$ ) of at least 50 seeds were recorded for each biological replicates, and three biological replicates were done for calculating germination rates (B).

Post-germination growth of the seedlings under abiotic stresses, and shoot and root lengths were recorded. Bars show standard deviations of three independent replicates. Asterisks indicate significant differences between the wild type and the overexpression lines evaluated with $S t u d e n t ' s t$-test $\left({ }^{\star} P<0.05\right.$, $\left.{ }^{\star \star} P<0.01\right)$. 
A

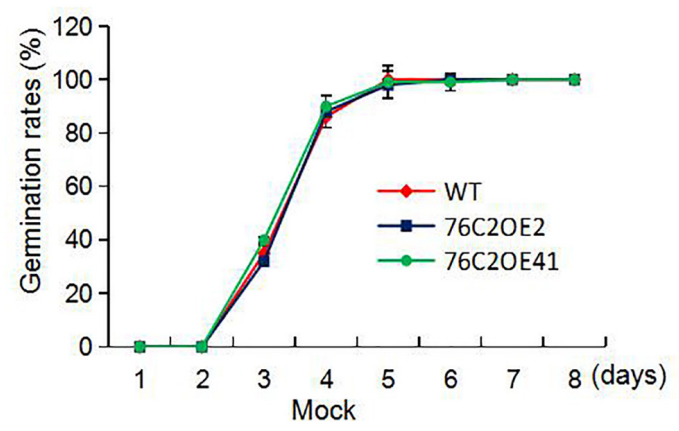

B

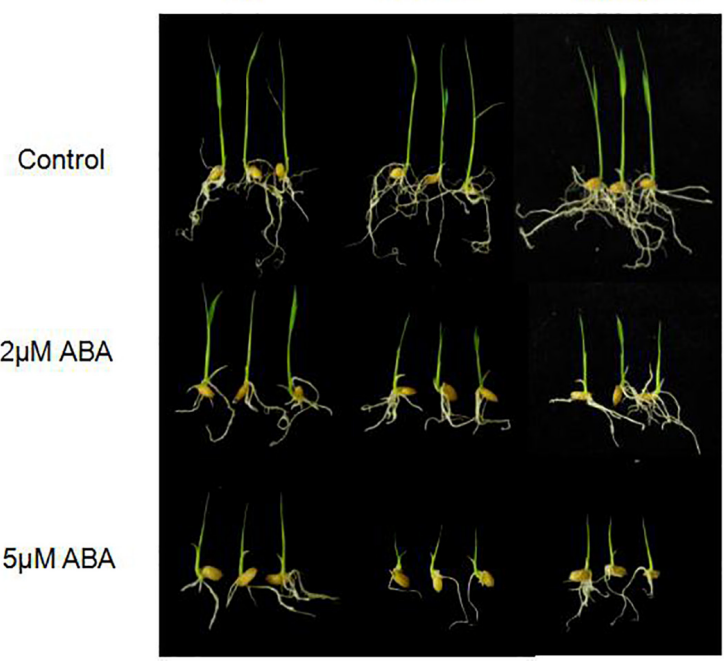

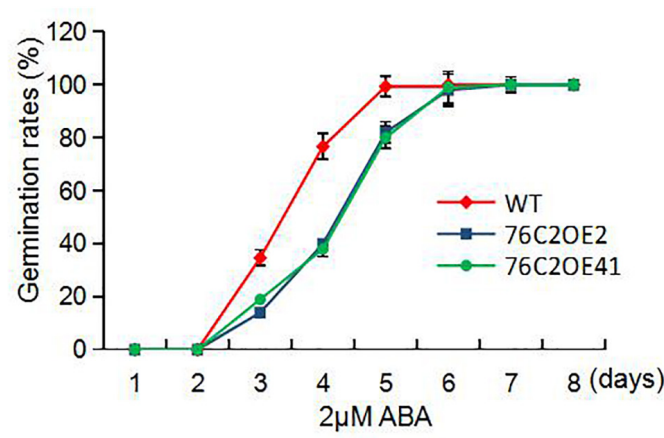

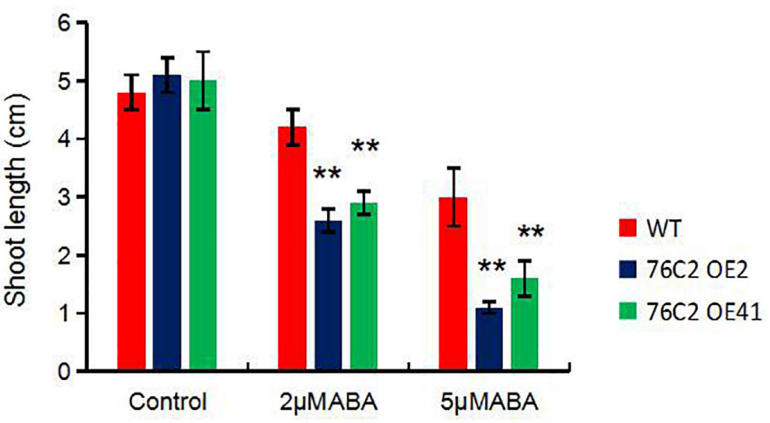

FIGURE 3 | Overexpression of AtUGT76C2 increased the plant sensitivity to abscisic acid (ABA) during germination and post-germination growth. (A) Rice seeds were imbibed at $28^{\circ} \mathrm{C}$ in hydroponic solution with $2 \mu \mathrm{M}$ ABA (dissolved with little ethonal) and without ABA (Mock, with equal amount of ethonal). Germination (based on radicles $>2 \mathrm{~mm}$ ) of at least 50 seeds were recorded for each biological replicates, and three biological replicates were done for calculating germination rates (B). Post-germination growth of the seedlings under ABA, and shoot lengths were recorded. Bars show standard deviations of three independent replicates. Asterisks indicate significant differences between the wild type and the overexpression lines evaluated with Student's $t$-test $\left({ }^{* *} P<0.01\right)$.

rice could catalyze the glycosylation of cytokinin. In addition, we also evaluated the endogenous cytokinin content in the three plants and found that it decreased in the two UGT76C2 overexpression lines (Figure 1D), demonstrating that UGT76C2 affected cytokinin homeostasis in transgenic rice.

\section{Overexpression of the AtUGT76C2 in Rice Leads to Sensitivity to Abiotic Stresses and ABA at Germination and Postgermination Stages}

To see the performance of the UGT76C2 transgenic lines in response to abiotic stresses, seeds of wild type, UGT76C2OE2, and UGT76C2OE41 were exposed to $100 \mathrm{mM} \mathrm{NaCl}, 150 \mathrm{mM}$ mannitol, and $7.5 \%$ PEG for germination, and the germination rates were recorded in the following 8 days. As illustrated in Figure 2A, OE2 and OE41 showed lower germination rates compared to WT under these adverse conditions. The subsequent growth of OE2 and OE41 seedlings was also more affected under abiotic stresses. The two OE lines were observed to have shorter shoots and roots than that of WT when kept growing under mannitol, $\mathrm{NaCl}$, and PEG conditions for 1 week more after germination. Especially the shoot growth of 76C2OE lines is significantly inhibited under PEG and $\mathrm{NaCl}$ treatments (Figure 2B). Next, we also examined the response of UGT76C2 overexpression lines upon ABA treatment in terms of seed germination and shoot growth. It was observed that OE2 and OE41 exhibited lower germination rates as well as slower shoot growth (Figure 3).

\section{Expression of AtUGT76C2 Improves Stress Adaptation and Enhances Root Growth at Seedling Stage}

To see the performance of AtUGT76C2 overexpression plants under salt stress, the soil-growing 2-week-old WT, 

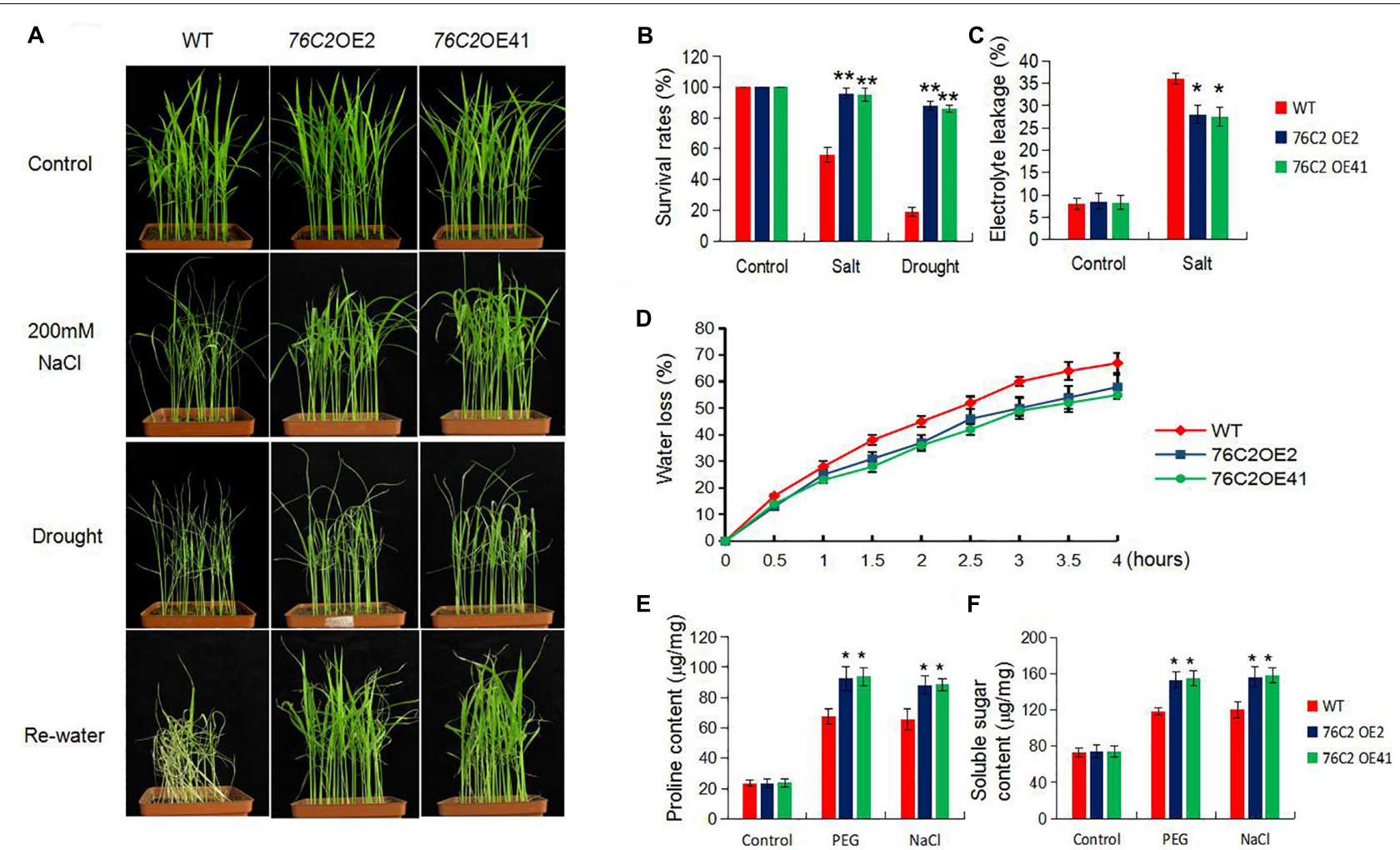

FIGURE 4 | Overexpression of AtUGT76C2 in rice enhanced salt and drought tolerance in the adult transgenic plants. (A) For salt treatment, 2-week-old rice seedlings were exposed to $200 \mathrm{mM} \mathrm{NaCl}$ for 7 days. For drought treatment, the plants were dried for 1 week, and then allowed for recovery for 7 days. (B) Survival rates of the transgenic and the control rice after salt and drought treatment. (C) Electrolyte leakage of the plants after salt treatment. (D) Water loss rate of detached leaves during $4 \mathrm{~h}$. Proline (E) and soluble sugar (F) content under PEG and $\mathrm{NaCl}$ treatments. Each assay was done at three independent biological replicates. Asterisks indicate significant differences between the wild type and the overexpression lines evaluated with Student's $t$-test $\left({ }^{\star} P<0.05\right.$, $\left.{ }^{\star *} P<0.01\right)$.

UGT76C2OE2, and UGT76C2OE41 seedlings were exposed to $200 \mathrm{mM} \mathrm{NaCl}$ for 2 weeks, and then the WT plants became withered, while the transgenic lines were more tolerant to salt stress (Figure 4A). Their tolerance to salt stress was also assessed in terms of survival rate and electrolyte leakage. The survival rates of WT, OE2, and OE41 were 53.6, 93.3, and $92.1 \%$, respectively (Figure $4 \mathbf{B}$ ), and the two transgenic lines OE2 and OE41 exhibited less electrolyte leakage under salt stress than WT, indicating that less damage is caused in the UGT76C2 overexpression plants (Figure 4C). For drought stress treatment, 2-week-old WT, OE2, and OE41 were dried for 7 days and then re-irrigated. It was observed that most of the WT plants wilted (Figure 4A), while the two OE lines were still vigorous and showed higher survival rates, 86.2 for $76 \mathrm{C} 2 \mathrm{OE} 2$ and $85.3 \%$ for $76 \mathrm{C} 2 \mathrm{OE} 41$, respectively. However, the survival rate of WT plants was only $18.8 \%$ (Figure $4 B$ ). To make a further investigation, we also detected the water loss rates of the detached leaves from the three plants in $4 \mathrm{~h}$. The result indicates that the two transgenic rice lines exhibited lower water loss than that of WT. After $4 \mathrm{~h}$, the water loss rates of WT, OE2, and OE41 were 67.1, 58.0, and 55.2\%, respectively (Figure 4D). Additionally, the proline and soluble sugars, which serve as osmotic regulators, were also accumulated more in OE2 and OE41 plants (Figures 4E,F). Above all, from both visual phenotype and assessment of physiological indexes, drought and salt stress tolerance is greatly improved in UGT76C2OE rice plants.

Interestingly, it was also observed that, with the growth of the rice seedlings, root growth was enhanced upon UGT76C2 overexpression in the transgenic lines. For seedlings as young as 4 days old, they showed little difference in root length. However, for 7, 10, and 15-days-old seedlings, the roots of the UGT76C2 overexpression lines were obviously longer than those of WT (Figure 5), which also contributes to the drought and salt stress tolerance of the UGT76C2 overexpression lines.

\section{Overexpression of AtUGT76C2 in Rice Reduced Stomatal Opening in Response to Drought Stress}

When suffering from drought stress, plants will soon reduce the transpiration rate and water loss, for example, by controlling stomata movement. Therefore, we compared the stomatal opening of 4-week-old rice leaves of WT, UGT76C2OE2, and UGT76C2OE41. The degrees of stomatal opening are shown in Figure 6A. Under the non-treatment condition, the proportion of closed stomata showed a little difference in WT and transgenic plants at $20.2,17$, and $18.7 \%$ for WT, OE2, and 

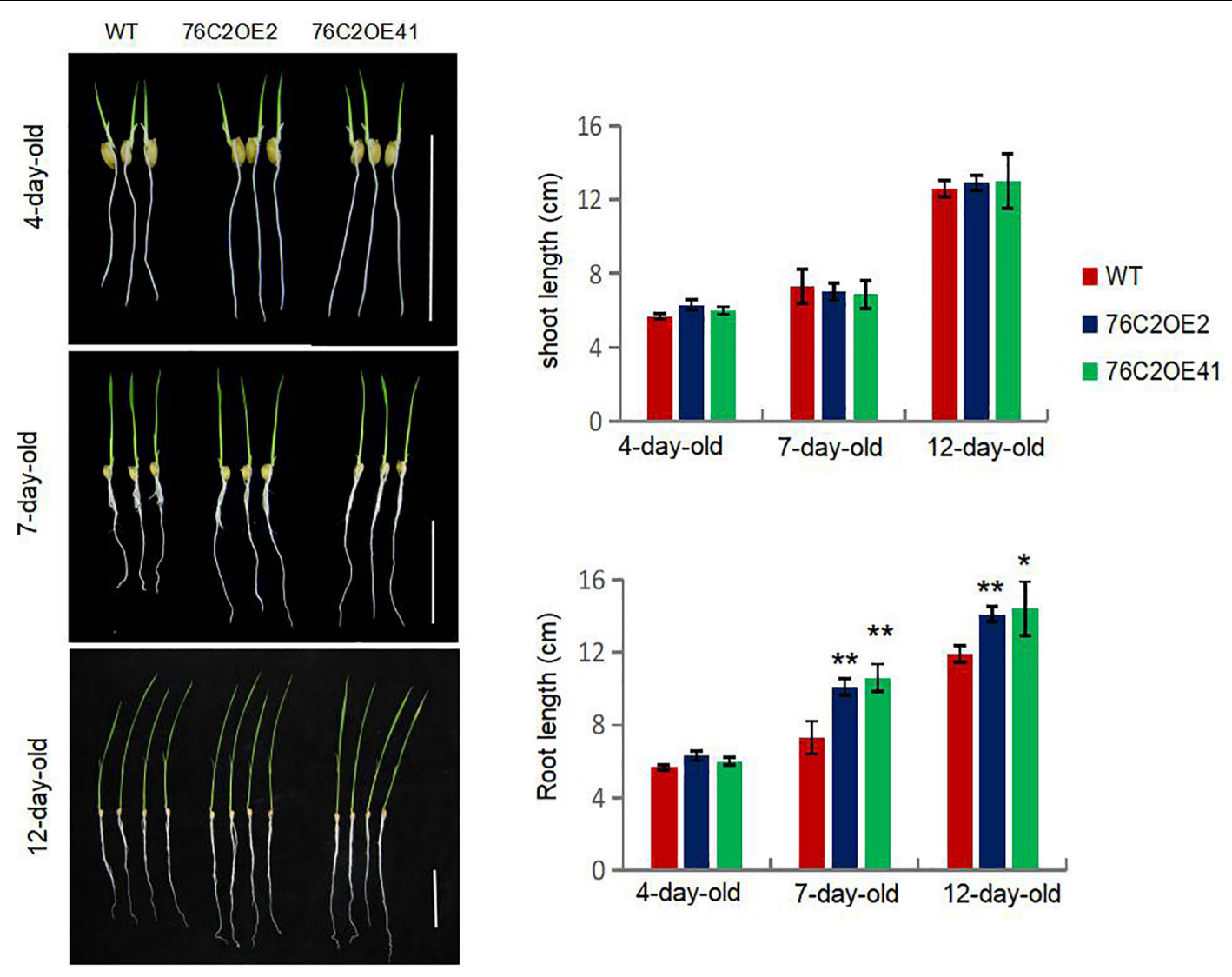

FIGURE 5 | Overexpression of AtUGT76C2 in rice enhanced root growth (bar $=5 \mathrm{~cm}$ ). Bars show standard deviations of at least 10 seedlings. Asterisks indicate significant differences between wild type and the overexpression lines evaluated with Student's $t$-test ( ${ }^{\star} P<0.05$, ${ }^{\star \star} P<0.01$ ).

OE41, respectively. However, the proportion of completely open stomata was more in WT than in OE plants and that of partially open stomata was more in OE than in WT. After exposing the detached leaves in open air for $2 \mathrm{~h}$, the number of closed stomata increased and that of completely open stomata substantially decreased. More completely closed stomata and less completely/partially open stomata were observed in OE2 and OE41 leaves compared with WT. The proportion of completely closed stomata accounts for 28.4, 48.8, and 46.5\% for WT, $\mathrm{OE} 2$, and OE41, respectively. The completely open stomata for WT, OE2, and OE41 plants account for 64, 47.2, and 49.6\%, respectively, and the partially open stomata were $7.6,3.9$, and $4 \%$ for WT, OE2, and OE41 (Figure 6B). These results suggested that the elevated water saving capacity and the drought resistance in AtUGT76C2 transgenic rice were largely due to reduced stomatal opening.

Stomatal movement is known to be closely related to ABA level or ABA signaling. Here we determined the ABA contents in WT and UGT76C2OE plants under normal and drought stress conditions. It was observed that the endogenous ABA levels in both AtUGT76C2 overexpression and WT seedlings were more accumulated in response to drought stress; however, we did not see any significant differences in ABA levels between transgenic and WT seedlings under both normal and drought stress conditions (Figure 6C). These results imply that UGT76C2 overexpression might have affected ABA signaling instead of ABA synthesis.

\section{Overexpression of AtUGT76C2 Enhanced ROS Scavenging in Transgenic Plants}

It is known to all that stresses cause an accumulation of reactive oxygen species (ROS) that can damage the membrane systems. Here, we also investigated ROS levels in UGT76C2OE and WT plant under stress condition. DAB and NBT staining indicated that ROS accumulated more in WT than in UGT76C2 transgenic lines (Figure 7). To explain the differential ROS production, we firstly examined the expression of genes encoding ROS scavenging enzymes, such as catalase isozyme A (CAT-A), catalase isozyme B (CAT-B), ascorbate peroxidase 2 (APX2), iron-superoxide dismutase $\mathrm{b}$ (Fe-SODb), and copper/zincsuperoxide dismutase (SODCc2). Quantitative RT-PCR showed that all the five genes were upregulated by salinity and drought and were more upregulated in the AtUGT76C2 transgenic lines (Figure 8A). Besides, we also examined the antioxidant enzyme activities of CAT, APX and SOD in WT and transgenic plant under normal and stress conditions, and found that they were higher in transgenic lines than in WT in response to 


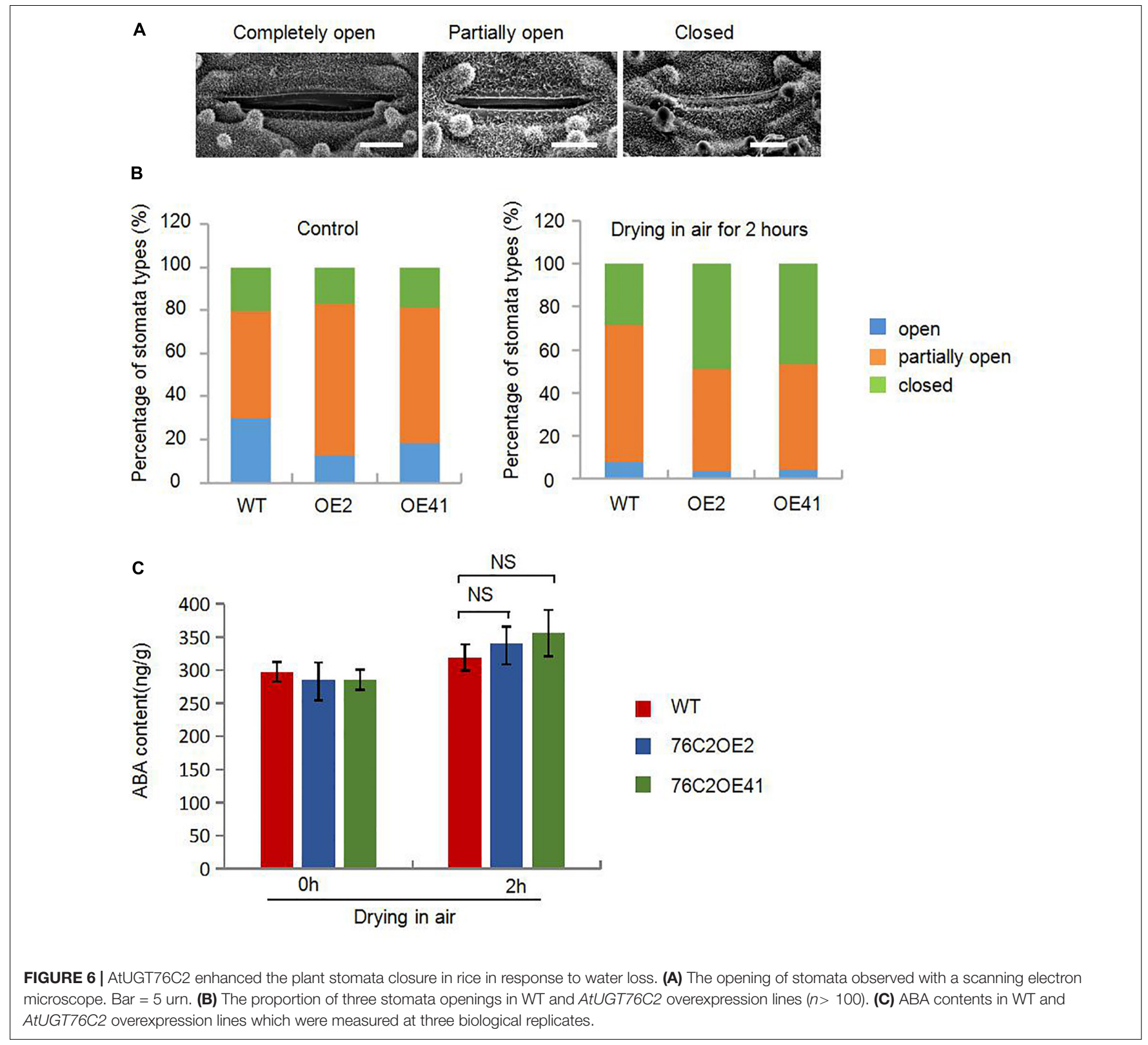

abiotic stresses, whereas showed no difference under control condition (Figure 8B).

\section{Expressions of Stress-Related Genes Were Affected Upon UGT76C2 Overexpression in Rice}

To investigate the potential molecular pathways affected by UGT76C2 in regulating stress tolerance, we then monitored the expressions of eight abiotic stress-related genes including OsSOS1, OsPIP2.1, OsDREB2A, OsCOIN, OsABF2, OsRAB16, OsP5CR, and OsP5CS1. Two-week-old wild type and UGT76C2OE plants were exposed to $\mathrm{NaCl}$ and $\mathrm{PEG}$, respectively, for $12 \mathrm{~h}$, and $\mathrm{qPCR}$ was performed. The results showed that all these genes were induced at different degrees by salt and drought and were more highly expressed in the overexpression lines compared with that in WT plants (Figure 9). For instance, in response to salt stress, the expression of OsSOS1, OsPIP2.1, and OsRAB16 were significantly induced, more than 15 -folds in transgenic lines, while less induced in WT plants. Upon exposure to PEG, DREB2A was substantially elevated in the two overexpression lines, up to 25 -fold, while less than 20 -fold in WT plants. The differential expression of these stress-regulated genes also accounted for the stress tolerance of $76 \mathrm{C} 2 \mathrm{OE}$ plants.

\section{DISCUSSION}

Rice is the most consumed staple food in the world that feeds more than half of the world's population. However, with the 


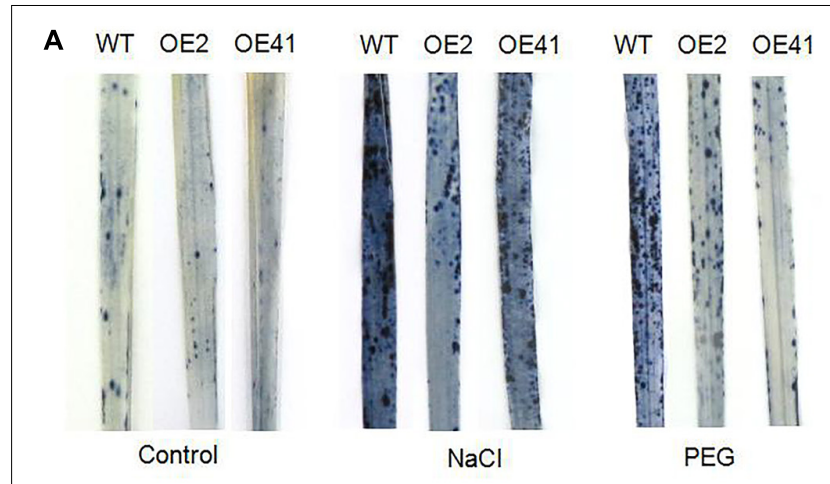

B

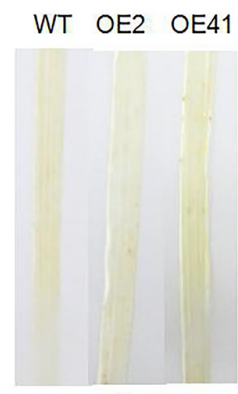

Control

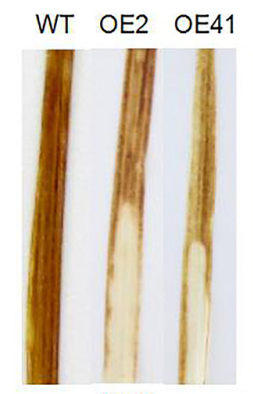

$\mathrm{NaCl}$

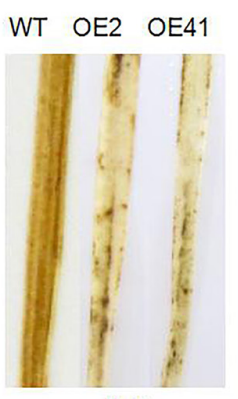

PEG
C

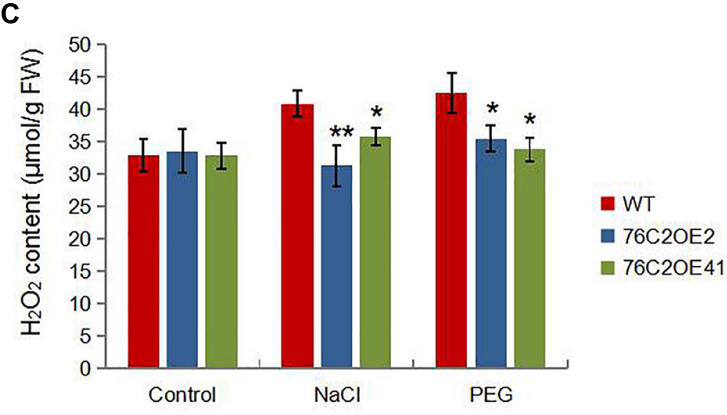

FIGURE $7 \mid$ ROS production in the transgenic plants in response to salt and drought conditions. Four-week-old WT and AtUGT76C2 overexpression rice plants were subjected to $200 \mathrm{mM} \mathrm{NaCl}$ and 15\% PEG8000 treatments for $12 \mathrm{~h}$, respectively. Then rice leaves were subjected to NBT (A) and DAB staining (B), and $\mathrm{H}_{2} \mathrm{O}_{2}$ contents were quantitatively measured (C). For each staining and $\mathrm{H}_{2} \mathrm{O}_{2}$ determination, leaves were harvested from at least five independent plants, and the pictures were representative of most samples. Asterisks indicate significant differences between wild type and the overexpression lines evaluated with Student's $t$-test $\left({ }^{*} P<0.05\right.$, $\left.{ }^{\star \star} P<0.01\right)$.

deterioration of the current global environment and uneven precipitation, the area of soil drying is gradually increasing. As a semi-aquatic crop, rice is greatly threatened by drought and salt stresses. In order to cultivate rice varieties with good traits, it is important to explore candidate genes that can be applied into rice breeding. To cope with adverse environmental stimuli, scientists are devoted to identifying stress-responsive genes and generate stress-adaptive crops by molecular breeding or gene engineering. Adverse environmental factors affect many aspects of plants but will finally lead to disturbance of the metabolic process that closely associated with plant growth and development. UDP-glycosyltransferases, as the manager of cellular homeostasis in plants (Lim and Bowles, 2004; Bowles et al., 2005), likely provide new strategies in cultivating stressresistant crops.

In this study, we introduced the Arabidopsis UGT76C2 into rice, which affects cytokinin homeostasis (Figure 1) and enhances stress tolerance in the transgenic rice (Figure 4). We demonstrate that UGT76C2 contributes to stress tolerance in several aspects. First, overexpression of UGT76C2 decreased the level of cytokinins (Figure 1D), which is generally regarded as a negative factor in plant abiotic responses (Nishiyama et al., 2011). UGT76C2 overexpression also enhanced root growth in the transgenic lines (Figure 5). This finding is consistent with several former studies indicating the role of cytokinin in modulating root development (Zalabák et al., 2013). For instance, overexpression of $C K X$ in barley led to cytokinin deficiency, which enlarged the root system and increased root biomass (Zalewski et al., 2010). Pineda Rodó et al. (2008) found that constitutive expression of a zeatin-O-glycosyltransferase in maize resulted in more branched roots with greater biomass. A former study in our group also reported that overexpression of UGT76C2 enhanced the lateral root density in Arabidopsis (Wang et al., 2011). A strong root system is a critical trait in adapting to water deficit. A recent study found that the ZmPTF1 transcription factor enhanced drought tolerance by promoting root growth (Li et al., 2019). Additionally, according to Li et al.'s (2018), overexpression of $R C c 3$ in rice increased the root system and enhanced salt tolerance. Thus, it is believed that enhanced root growth in UGT76C2 overexpression lines greatly contribute to plant tolerance to drought and salt.

It is well known that abiotic stresses cause an excess production of reactive oxygen species. Cellular ROS are usually maintained at a relatively low level through a wide range of scavenging and detoxification mechanisms. More and more studies have identified the connection between cytokinin and oxidative stress. Wang et al. (2015) showed that the overproduction of cytokinin by overexpressing AtIPT8 increased ROS production and decreased the CAT and POD activity in response to salt stress, which thus declined salt stress resistance. Additionally, Zwack and Rashotte (2015) showed that cytokinin response factor 6 functions in mediating oxidative stress response via attenuating cytokinin signaling. In our study, we found that overexpression of $A t U G T 76 C 2$ in rice, which decreased cytokinin level, resulted in less ROS accumulation under drought and salt stresses. Furthermore, the transcripts of ROS-scavenging enzymes such as CAT, SOD, and POD were upregulated, and their enzyme activities were also enhanced upon UGT76C2 overexpression (Figure 8). These findings further suggest that cytokinin plays a negative role for the plant to cope with oxidative stress, which is in agreement with former studies (Wang et al., 2015; Zwack and Rashotte, 2015).

We also found that the ectopic expression of UGT76C2 impeded seed germination and postgermination growth under drought and salt stress (Figures 2, 3) while improving stress tolerance in transgenic rice seedlings (Figure 4), suggesting that the role of $A t U G T 76 C 2$ is likely related to ABA signaling. ABA plays pivotal roles in response to abiotic stresses. When the plants are challenged by water deficit, ABA is soon synthesized 

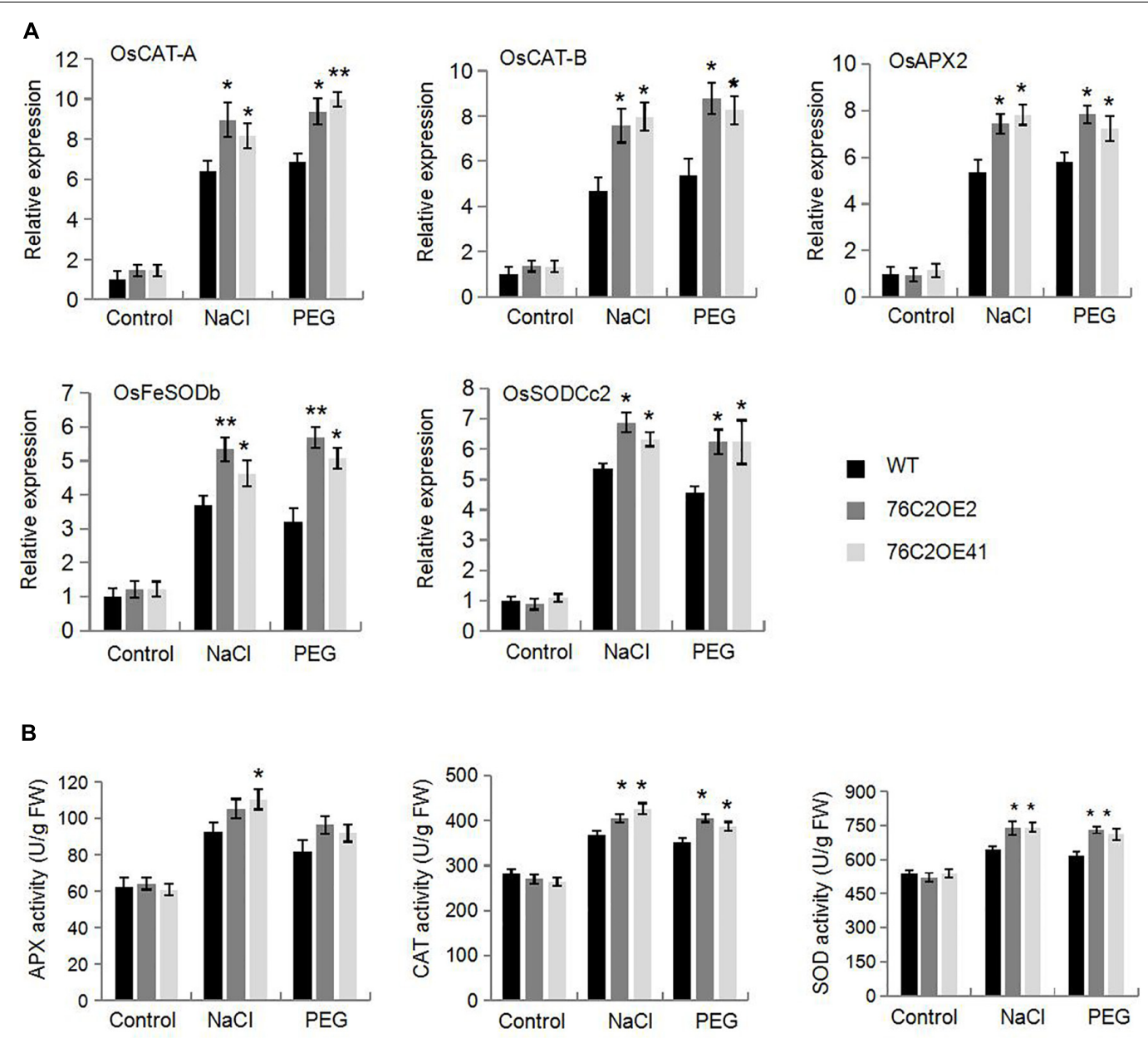

FIGURE 8 | Overexpression of AtUGT76C2 enhanced ROS scavenging in the transgenic plants. Four-week-old WT and AtUGT76C2 overexpression rice plants were subjected to $200 \mathrm{mM} \mathrm{NaCl}$ and $250 \mathrm{mM}$ PEG treatments for $12 \mathrm{~h}$, respectively. Then the transcript levels (A) and activities of ROS scavenging enzymes (B) were determined. These experiments were done at three independent biological replicates. Asterisks indicate significant differences between the wild type and the overexpression lines evaluated with Student's $t$-test $\left({ }^{\star} P<0.05,{ }^{* *} P<0.01\right)$.

and triggers the expression of many ABA-dependent genes in coping with stresses. During the germination and the postgermination stages, ABA helps to arrest the germination and the postgermination growth, which is actually a protective mechanism to young seedlings (Lopez-Molina et al., 2002). After the plants grow up into adult plants, ABA on one side contributes to induce stomatal closure in response to drought stress and on the other side activates the expression of some stressresponsive genes to cope with adverse conditions. Consistently, in our study, more closed stomata were observed in UGT76C2overexpressed rice than in WT plants when confronting drought stress (Figure 6B). Although the ABA level was not affected in $U G T 76 C 2 \mathrm{OE}$ plants in response to dehydration (Figure $6 \mathrm{C}$ ), it is speculated that $\mathrm{ABA}$ signaling is enhanced. This point could also be approved by the upregulation of ABA signaling or responsive genes in $U G T 76 C 2 \mathrm{OE}$ plants. For instance, OsABF2 and OsRAB16 are positive regulators in ABA signaling pathway (Lu et al., 2009; Hossain et al., 2010), and both genes were more upregulated upon UGT76C2 overexpression in response to drought and salt. OsPIP2.1 is strongly induced by ABA, and PIP2.1 activation was required for stomatal closure in response to ABA treatment in Arabidopsis (Rodrigues et al., 2017; Ding et al., 2019). OsCOIN is induced by ABA as well as cold, salt, and drought stresses. The overexpression of OsCOIN could increase tolerance to chilling, salt, and drought and enhance proline level in rice (Liu et al., 2007). The expression of the proline biosynthesis genes OsP5CS1 and OsP5CR, which are responsive to osmotic and salt stresses and ABA (Hu et al., 1992; Sripinyowanich et al., 2013), was also upregulated in UGT76C2 overexpressors under salt and drought (Figure 9).

UGT76C2 recognizes all cytokinins and modifies the hormones at the N7 and N9 positions (Hou et al., 2004). The cytokinin glucosides are generally regarded as biologically inactive CK forms in CK signaling (Hothorn et al., 2011; Lomin et al., 2015). Interestingly, there is evidence indicating that the N-linked (N6 and/or N9) cytokinin glycosides show antisenescent and antioxidant activity (Hönig et al., 2018). The effects might be explained by the presence of electronegative 

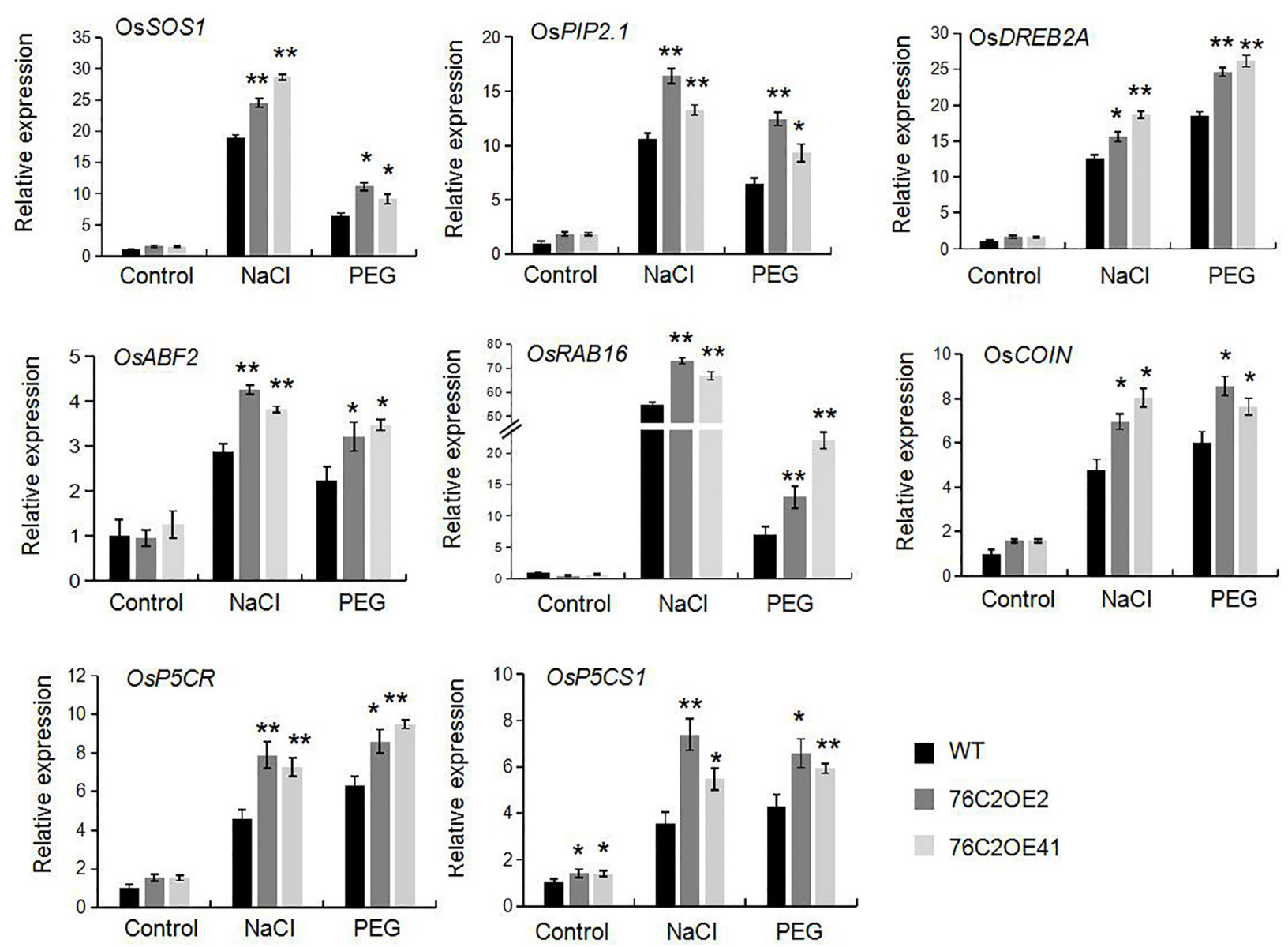

FIGURE 9 | Expression of abiotic stress related genes in wild type, UGT76C2 overexpression plants in response to stresses. The experiments included three biological replicates, each with three technical replicates. Asterisks indicate significant differences between the wild type and the overexpression lines evaluated with Student's $t$-test $\left({ }^{\star} P<0.05,{ }^{\star \star} P<0.01\right)$.

atoms, which are near the N6 and/or N9 atoms of purine (Hönig et al., 2018). A recent study also reported that some types of N-linked CK glucosides, such as transzeatin (tZ) N7and N9-glucosides, efficiently release free CK bases that are probably responsible for the biological activities (Hoyerová and Hošek, 2020). Additionally, in a latest study, Hallmark et al. (2020) reported that the exogenous application of trans-Zeatin$\mathrm{N}$-glucosides could lead to CK response. All these findings updated our understanding in the role of cytokinin glycosides. It can be inferred that the heterologous expression of Arabidopsis UGT76C2 into rice might also cause some other effects in the biochemical and molecular levels that are beyond our study here. Furthermore, cytokinin signaling under abiotic stress is acting as an inter-cellular communication network, which is essential to crosstalk with other types of phytohormones except ABA. It was demonstrated that cytokinin and auxin play complementary actions in regulating a series of plant developmental processes, which also work together in plant response to stresses (Bielach et al., 2017). A link also exists between cytokinin and SA signaling pathway via ARR2 and TGA3 in stress response (Verma et al., 2016). From these points of view, cytokinin-regulated stress response is far more complicated than we have revealed here. The detailed mechanisms remain elusive and require further investigation for more insights into cytokinin involvement in plant defense systems. Anyway, however, we reveal that the overexpression of UGT76C2 in rice brings good traits in coping with stresses, which lays a theoretical foundation that UGT76C2 could serve as a promising candidate gene for cultivating salineand drought-tolerant crops.

\section{DATA AVAILABILITY STATEMENT}

The raw data supporting the conclusions of this article will be made available by the authors, without undue reservation.

\section{AUTHOR CONTRIBUTIONS}

YL, FL, and PL carried out the experiments. YL, TW, and $\mathrm{CZ}$ analyzed the data. YL and $\mathrm{BH}$ conceived and designed the research. YL and FL wrote the manuscript. All authors read and approved the final manuscript. 


\section{FUNDING}

This research was supported by the Key R\&D Program Projects in Shandong Province (No. 2018GNC110019) and the National Natural Science Foundation of China (No. 31970290).

\section{REFERENCES}

Bielach, A., Hrtyan, M., and Tognetti, V. B. (2017). Plants under stress: involvement of auxin and cytokinin. Int. J. Mol. Sci. 18:1427. doi: 10.3390/ijms18071427

Bowles, D., Isayenkova, J., Lim, E. K., and Poppenberger, B. (2005). Glycosyltransferases: managers of small molecules. Curr. Opin. Plant Biol. 8, 254-263. doi: 10.1016/j.pbi.2005.03.007

Bowles, D., Lim, E.-K., Poppenberger, B., and Vaistij, F. E. (2006). Glycosyltransferases of lipophilic small molecules. Annu. Rev. Plant Biol. 57, 567-597. doi: 10.1146/annurev.arplant.57.032905.105429

Cai, W., Liu, W., Wang, W. S., Fu, Z. W., Han, T. T., and Lu, Y. T. (2015). Overexpression of rat neurons nitric oxide synthase in rice enhances drought and salt tolerance. PLoS One 10:e0131599. doi: 10.1371/journal.pone.013 1599

Chen, M., Zhao, Y., Zhuo, C., Lu, S., and Guo, Z. (2015). Overexpression of a NFYC transcription factor from bermudagrass confers tolerance to drought and salinity in transgenic rice. Plant Biotechnol. J. 13, 482-491. doi: 10.1111/pbi. 12270

Cortleven, A., Leuendorf, J. E., Frank, M., Pezzetta, D., Bolt, S., and Schmülling, T. (2019). Cytokinin action in response to abiotic and biotic stresses in plants. Plant Cell Environ. 42, 998-1018. doi: 10.1111/pce.13494

Ding, L., Uehlein, N., Kaldenhoff, R., Guo, S., Zhu, Y., and Kai, L. (2019). Aquaporin PIP2;1 affects water transport and root growth in rice (Oryza sativa L.). Plant Physiol. Biochem. 139, 152-160. doi: 10.1016/j.plaphy.2019.03.017

Du, Y. Y., Wang, P. C., Chen, J., and Song, C. P. (2008). Comprehensive functional analysis of the catalase gene family in Arabidopsis thaliana. Plant Physiol. 050, 1318-1326. doi: 10.1111/j.1744-7909.2008.00741.x

El-Showk, S., Ruonala, R., and Helariutta, Y. (2013). Crossing paths: cytokinin signalling and crosstalk. Development 140, 1373-1383. doi: 10.1242/dev.086371

Hai, N. N., Chuong, N. N., Tu, N. H. C., Kisiala, A., Hoang, X. L. T., and Thao, N. P. (2020). Role and regulation of cytokinins in plant response to drought stress. Plants 9:422. doi: 10.3390/plants9040422

Hallmark, H. T., Černý, M., Brzobohatý, B., and Rashotte, A. M. (2020). transZeatin-N-glucosides have biological activity in Arabidopsis thaliana. PLoS One 15:e0232762. doi: 10.1371/journal.pone.0232762

Hansen, H., and Dörffling, K. (2003). Root-derived trans-zeatin riboside and abscisic acid in drought-stressed and rewatered sunflower plants: interaction in the control of leaf diffusive resistance? Funct. Plant Biol. 30, 365-375. doi: 10.1071/FP02223

Hönig, M., Plíhalová, L., Husičková, A., Nisler, J., and Doležal, K. (2018). Role of cytokinins in senescence, antioxidant defence and photosynthesis. Int. J. Mol Sci. 19:4045. doi: 10.3390/ijms19124045

Hossain, M. A., Cho, J. I., Han, M., Ahn, C. H., Jeon, J. S., An, G., et al. (2010). The ABRE-binding bZIP transcription factor OsABF2 is a positive regulator of abiotic stress and ABA signaling in rice. J. Plant Physiol. 167, 1512-1520. doi: 10.1016/j.jplph.2010.05.008

Hothorn, M., Dabi, T., and Chory, J. (2011). Structural basis for cytokinin recognition by Arabidopsis thaliana histidine kinase 4. Nat. Chem. Biol. 7, 766-768. doi: 10.1038/nchembio.667

Hou, B., Lim, E. K., Higgins, G. S., and Bowles, D. J. (2004). N-glucosylation of cytokinins by glycosyltransferases of Arabidopsis thaliana. J. Biol. Chem. 279, 47822-47832. doi: 10.1074/jbc.M409569200

Hoyerová, K., and Hošek, P. (2020). New insights into the metabolism and role of cytokinin N-glucosides in plants. Front. Plant Sci. 11:741. doi: 10.3389/fpls. 2020.00741

Hu, C. A., Delauney, A. J., and Verma, D. P. (1992). A bifunctional enzyme (delta 1-pyrroline-5-carboxylate synthetase) catalyzes the first two steps in proline biosynthesis in plants. Proc. Natl. Acad. Sci. U.S.A. 89, 9354-9358. doi: 10.1073/ pnas.89.19.9354

\section{SUPPLEMENTARY MATERIAL}

The Supplementary Material for this article can be found online at: https://www.frontiersin.org/articles/10.3389/fpls.2020. 560696/full\#supplementary-material

Huang, X., Hou, L., Meng, J., You, H., Li, Z., Gong, Z., et al. (2018). The antagonistic action of abscisic acid and cytokinin signaling mediates drought stress response in Arabidopsis. Mol .Plant. 11, 970-982. doi: 10.1016/j.molp.2018.05.001

Jeon, J., Kim, N. Y., Kim, S., Kang, N. Y., Novák, O., Ku, S. J., et al. (2010). A subset of cytokinin two-component signaling system plays a role in cold temperature stress response in Arabidopsis. J. Biol. Chem. 285, 23371-23386. doi: 10.1074/jbc.M109.096644

Kang, J., Li, J., Gao, S., Tian, C., and Zha, X. (2017). Overexpression of the leucinerich receptor-like kinase gene LRK2 increases drought tolerance and tiller number in rice. Plant Biotechnol. J. 15, 1175-1185. doi: 10.1111/pbi.12707

Kang, N. Y., Cho, C., Kim, N. Y., and Kim, J. (2012). Cytokinin receptor-dependent and receptor-independent pathways in the dehydration response of Arabidopsis thaliana. J. Plant Physiol. 169, 1382-1391. doi: 10.1016/j.jplph.2012.05.007

Kuppu, S., Mishra, N., Hu, R., Sun, L., Zhu, X., Shen, G., et al. (2013). Water-deficit inducible expression of a cytokinin biosynthetic gene IPT improves drought tolerance in cotton. PLoS One 8:e64190. doi: 10.1371/journal.pone.0064190

Lairson, L. L., Henrissat, B., Davies, G. J., and Withers, S. G. (2008). Glycosyltransferases: structures, functions, and mechanisms. Annu. Rev. Biochem. 77, 521-555. doi: 10.1146/annurev.biochem.76.061005.092322

Leta, T. B., Miccah, S. S., Steven, M. R., Wondyifraw, T., and Francis, W. (2016). Drought tolerant tropical maize (Zea mays L.) developed through genetic transformation with isopentenyltransferase gene. Afr. J. Biotechnol. 15, 24472464. doi: 10.5897/AJB2016.15228

Li, W., Li, M., Zhang, W., Welti, R., and Wang, X. (2004). The plasma membranebound phospholipase $\mathrm{D} \delta$ enhances freezing tolerance in Arabidopsis thaliana. Nat. Biotechnol. 22, 427-433. doi: 10.1038/nbt949

Li, X., Chen, R., Chu, Y., Huang, J., Jin, L., Wang, G., et al. (2018). Overexpression of RCc3 improves root system architecture and enhances salt tolerance in rice. Plant Physiol. Biochem. 130, 566-576. doi: 10.1016/j.plaphy.2018.08.008

Li, X., Michlmayr, H., Schweiger, W., Malachova, A., Shin, S., Huang, Y., et al. (2017). A barley UDP-glucosyltransferase inactivates nivalenol and provides fusarium head blight resistance in transgenic wheat. J. Exp. Bot. 68, 2187-2197. doi: 10.1093/jxb/erx109

Li, Y. J., Wang, B., Dong, R. R., and Hou, B. K. (2015). AtUGT76C2, an Arabidopsis cytokinin glycosyltransferase is involved in drought stress adaptation. Plant Sci. 236, 157-167. doi: 10.1016/j.plantsci.2015.04.002

Li, Z., Liu, C., Zhang, Y., Wang, B., Ran, Q., and Zhang, J. (2019). The bHLH family member ZmPTF1 regulates drought tolerance in maize by promoting root development and abscisic acid synthesis. J. Exp. Bot. 70, 5471-5486. doi: 10.1093/jxb/erz307

Lim, E. K., and Bowles, D. J. (2004). A class of plant glycosyltransferases involved in cellular homeostasis. EMBO J. 23, 2915-2922. doi: 10.1038/sj.emboj.7600295

Liu, K., Wang, L., Xu, Y., Chen, N., Ma, Q., and Chong, L. K. (2007). Overexpression of OsCOIN, a putative cold inducible zinc finger protein, increased tolerance to chilling, salt and drought, and enhanced proline level in rice. Planta 226, 1007-1016. doi: 10.2307/23389762

Lomin, S. N., Krivosheev, D. M., Steklov, M. Y., Arkhipov, D. V., Osolodkin, D. I., Schmülling, T., et al. (2015). Plant membrane assays with cytokinin receptors underpin the unique role of free cytokinin bases as biologically active ligands. J. Exp. Bot. 66, 1851-1863. doi: 10.1093/jxb/eru522

Lopez-Molina, L., Mongrand, S. B., Mclachlin, D. T., Chait, B. T., and Chua, N. H. (2002). ABI5 acts downstream of ABI3 to execute an ABA-dependent growth arrest during germination. Plant J. 32, 317-328. doi: 10.1046/j.1365-313X.2002. 01430.x

Lu, G., Gao, C., Zheng, X., and Han, B. (2009). Identification of OsbZIP72 as a positive regulator of ABA response and drought tolerance in rice. Planta 229, 605-615. doi: 10.1007/s00425-008-0857-3

Macková, H., Hronková, M., Dobrá, J., Turečková, V., Novák, O., Lubovská, Z., et al. (2013). Enhanced drought and heat stress tolerance of tobacco plants with 
ectopically enhanced cytokinin oxidase/dehydrogenase gene expression. J. Exp. Bot. 64, 2805-2815. doi: 10.1093/jxb/ert131

Mhamdi, A., Queval, G., Chaouch, S., Vanderauwera, S., Van Breusegem, F., and Noctor, G. (2010). Catalase function in plants: a focus on Arabidopsis mutants as stress-mimic models. J. Exp. Bot. 61, 4197-4220. doi: 10.1093/jxb/erq282

Nakazawa, K., Tanaka, H., and Arima, M. (1982). Rapid, simultaneous and sensitive determination of free hydroxyproline and proline in human serum by high-performance liquid chromatography. J. Chromatogr. Biomed. Appl. 233, 313-316. doi: 10.1016/S0378-4347(00)81759-4

Nishiyama, R., Watanabe, Y., Fujita, Y., Le, D. T., Kojima, M., Werner, T., et al. (2011). Analysis of cytokinin mutants and regulation of cytokinin metabolic genes reveals important regulatory roles of cytokinins in drought, salt and abscisic acid responses, and abscisic acid biosynthesis. Plant Cell. 23, 21692183. doi: $10.1105 /$ tpc. 111.087395

Pineda Rodó, A., Brugière, N., Vankova, R., Malbeck, J., Olson, J. M., Haines, S. C., et al. (2008). Over-expression of a zeatin O-glucosylation gene in maize leads to growth retardation and tasselseed formation. J. Exp. Bot. 59, 2673-2686. doi: $10.1093 / \mathrm{jxb} / \mathrm{ern} 137$

Raines, T., Blakley, I. C., Tsai, Y. C., Worthen, J. M., Franco-Zorrilla, J. M., Solano, R., et al. (2016). Characterization of the cytokinin-responsive transcriptome in rice. BMC Plant Biol. 16:260. doi: 10.1186/s12870-016-0932-z

Rodrigues, O., Reshetnyak, G., Grondin, A., Saijo, Y., Leonhardt, N., Maurel, C., et al. (2017). Aquaporins facilitate hydrogen peroxide entry into guard cells to mediate ABA- and pathogen-triggered stomatal closure. Proc. Natl. Acad. Sci. U.S.A. 114, 9200-9205. doi: 10.1073/pnas.1704754114

Shi, H., Ishitani, M., Kim, C., and Zhu, J. K. (2000). The Arabidopsis thaliana salt tolerance gene SOS1 encodes a putative $\mathrm{Na}+\mathrm{H}+$ antiporter. Proc. Natl. Acad. Sci. U.S.A. 97, 6896-6901. doi: 10.1073/pnas.120170197

Sripinyowanich, S., Klomsakul, P., Boonburapong, B., Bangyeekhun, T., Asami, T., $\mathrm{Gu}, \mathrm{H}$., et al. (2013). Exogenous ABA induces salt tolerance in indica rice (Oryza sativa L.): the role of OsP5CS1 and OsP5CR gene expression during salt stress. Environ. Exp. Bot. 86, 94-105. doi: 10.1016/j.envexpbot.2010.01.009

Sun, Y. G., Wang, B., Jin, S. H., Qu, X. X., Li, Y. J., and Hou, B. K. (2013). Ectopic expression of Arabidopsis glycosyltransferase UGT85A5 enhances salt stress tolerance in tobacco. PLoS One 8:e59924. doi: 10.1371/journal.pone.0059924

Tang, Y., Liu, K., Ju, Z., Li, X., Xu, K., Yi, Z., et al. (2017). JcDREB2, a physic Nut AP2/ERF gene, alters plant growth and salinity stress responses in transgenic rice. Front. Plant Sci. 8:306. doi: 10.3389/fpls.2017.00306

Tran, L. S., Urao, T., Qin, F., Maruyama, K., Kakimoto, T., Shinozaki, K., et al. (2007). Functional analysis of AHK1/ATHK1 and cytokinin receptor histidine kinases in response to abscisic acid, drought, and salt stress in Arabidopsis. Proc. Natl. Acad. Sci. U.S.A. 104, 20623-20628. doi: 10.1073/pnas.0706547105

Verma, D., Lakhanpal, N., and Singh, K. (2019). Genome-wide identification and characterization of abiotic-stress responsive SOD (superoxide dismutase) gene family in Brassica juncea and B. rapa. BMC Genomics. 20:227. doi: 10.1186/ s12864-019-5593-5

Verma, V., Ravindran, P., and Kumar, P. P. (2016). Plant hormone-mediated regulation of stress responses. BMC Plant Biol. 16:86. doi: 10.1186/s12870-016$0771-\mathrm{y}$
Wang, J., Ma, X. M., Kojima, M., Sakakibara, H., and Hou, B. K. (2011). $\mathrm{N}$-Glucosyltransferase UGT76C2 is Involved in cytokinin homeostasis and cytokinin response in Arabidopsis thaliana. Plant Cell Physiol. 52, 2200-2213. doi: $10.1093 / \mathrm{pcp} / \mathrm{pcr} 152$

Wang, Y., Shen, W., Chan, Z., and Wu, Y. (2015). Endogenous cytokinin overproduction modulates ROS homeostasis and decreases salt stress resistance in Arabidopsis thaliana. Front. Plant Sci. 6:1004. doi: 10.3389/fpls.2015. 01004

Wohlgemuth, H., Mittelstrass, K., Kschieschan, S., Bender, J., and Sandermann, H. J. (2002). Activation of an oxidative burst is a general feature of sensitive plants exposed to the air pollutant ozone. Plant Cell Environ. 25, 717-726. doi: 10.1046/j.1365-3040.2002.00859.x

Xiao, X. O., Zeng, Y. M., Cao, B. H., Lei, J. J., and Cheng, Y. J. (2017). PSAG12IPT overexpression in eggplant delays leaf senescence and induces abiotic stress tolerance. J. Hortic. Sci. Biotech. 92, 1-9. doi: 10.1080/14620316.2017.1287529

Xiong, H., Li, J., Liu, P., Duan, J., Zhao, Y., Guo, X., et al. (2014). Overexpression of OsMYB48-1, a novel MYB-related transcription factor, enhances drought and salinity tolerance in rice. PLoS One 9:e92913. doi: 10.1371/journal.pone. 0092913

Xu, Y., Burgess, P., Zhang, X., and Huang, B. (2016). Enhancing cytokinin synthesis by overexpressing ipt alleviated drought inhibition of root growth through activating ROS-scavenging systems in Agrostis stolonifera. J. Exp. Bot. 67, 1979-1992. doi: 10.1093/jxb/erw019

Zalabák, D., Pospíšlová, H., Šmehilová, M., Mrízová, K., Frébort, I., and Galuszka, P. (2013). Genetic engineering of cytokinin metabolism: prospective way to improve agricultural traits of crop plants. Biotechnol. Adv. 31, 97-117. doi: 10.1016/j.biotechadv.2011.12.003

Zalewski, W., Galuszka, P., Gasparis, S., Orczyk, W., and Nadolska-Orczyk, A. (2010). Silencing of the HvCKX1 gene decreases the cytokinin oxidase/dehydrogenase level in barley and leads to higher plant productivity. J. Exp. Bot. 61, 1839-1851. doi: 10.1093/jxb/erq052

Zhang, J., Schurr, U., and Davies, W. J. (1987). Control of stomatal behaviour by abscisic acid which apparently originates in the roots. J. Exp.Bot. 38, 1174-1181. doi: $10.1093 / \mathrm{jxb} / 38.7 .1174$

Zhu, J. K. (2002). Salt and drought stress signal transduction in plants. Annu. Rev. Plant Biol. 53, 247-273. doi: 10.1146/annurev.arplant.53.091401.143329

Zwack, P. J., and Rashotte, A. M. (2015). Interactions between cytokinin signalling and abiotic stress responses. J. Exp. Bot. 66, 4863-4871. doi: 10.1093/jxb/erv172

Conflict of Interest: The authors declare that the research was conducted in the absence of any commercial or financial relationships that could be construed as a potential conflict of interest.

Copyright $\odot 2020 \mathrm{Li}$, Liu, Li, Wang, Zheng and Hou. This is an open-access article distributed under the terms of the Creative Commons Attribution License (CC BY). The use, distribution or reproduction in other forums is permitted, provided the original author(s) and the copyright owner(s) are credited and that the original publication in this journal is cited, in accordance with accepted academic practice. No use, distribution or reproduction is permitted which does not comply with these terms. 